\title{
Accessibility analysis of multimodal transport systems using advanced GIS techniques
}

\author{
T. Vorraa \\ Citilabs Regional Director, Woking, UK
}

\begin{abstract}
This paper gives an overview of the English Department of Transport's advice and requirements regarding accessibility analysis as part of local transport plans. A GIS based software tool was developed to make it possible to meet these requirements and this tool is described and some examples of its use are given.
\end{abstract}

\section{Introduction}

The English Department for Transport (DfT) issued in beginning of the 2000s some guidance on accessibility planning for local authorities. The authorities are required to make accessibility planning part of their local transport plans. In the guidance, the DfT defines what accessibility planning is and why it's needed. Accessibility planning was introduced by a Social Exclusion Unit Report 'Making the Connections' (www.socialexclusion.gov.uk/downloaddoc.asp? $\mathrm{id}=66$ ). The report set out the relationship between transport, accessibility and social exclusion and a cross-Government strategy for improving access to jobs and essential public services.

Accessibility planning focuses on promoting social inclusion by tackling the accessibility problems experienced by those in disadvantaged groups and areas. These might include the availability, affordability and accessibility of local public transport, the design, location and delivery of non-transport services, and the ability of the community to reach those services by foot or cycle. It also focuses on access to those opportunities that are likely to have the most impact on life chances: employment, education, health care and food shops. The premise of accessibility planning is that policy development and service delivery can be improved to better meet the accessibility needs of local communities by being more evidence-led and through improved cross-sectoral working. 
Accessibility is not simply concerned with geographical barriers, but also covers cost, personal safety and security and other deterrents. The benefits of accessible public transport will not be fully realised unless people are able to reach it. Footway maintenance, the provision and design of road crossings and the position of street furniture are just some of the issues which also need to be addressed. An inaccessible or poorly planned pedestrian environment will be a major barrier for many potential users, including disabled and older people. Accessibility planning needs to consider the whole transport chain.

DfT contracted MVA, a UK based transport planning consultancy and part owner of Citilabs, to develop new methodologies for modelling accessibility to and through the multimodal transport system. This includes the ability to quickly measure accessibility to important public services. A GIS based software tool was developed as part of this project and MVA and Citilabs released this software under the name of 'Accession' in 2004.

\section{Modelling accessibility}

The underlying methodology in Accession enables users to undertake a range of accessibility assessments using each of the following measures of accessibility:

- Access measures

- Network measures

\subsection{Access measures}

The access measures are used to define the ease of access to/from the transport network. This measure is usually applied to public transport networks using a set of indicators:

Considering for example one of the potential indicators of social exclusion and rural bus accessibility indicators:

- $\quad$ Proportion of people within 10 minutes walk of a 5, 10, 15 minute bus service.

- Proportion of households in rural areas within a 10-13 minute walk of an hourly or better bus service.

This refers to access to/from the public transport network defined in terms of journey time/distance to/from an access point with user defined service frequencies for different user defined time periods and days of the week.

The methodology uses public transport mode, walk mode time/distance thresholds, public transport service frequency levels, time of day and day of week to undertake the accessibility assessment. As an example:

i. Public transport mode: Bus

ii. Vehicle type: Low floor bus

iii. Walk time: 10 minutes 
iv. Public transport service frequency level: At least 4 per hour v. Day of week: Monday-Friday vi. Time of day: AM peak

Access points that satisfy the above criteria (i, ii, iii, iv, v, vi) are then identified and these are treated as destinations for the subsequent walk routing process. Journey time/distance contours are subsequently produced (preceded by an interpolation stage). Accessibility reports and queries are separately to be used to determine the population groupings present within the defined journey time/distance threshold $\mathrm{C}$. The mathematical definition of the population within this threshold $\mathrm{C}$ is:

$$
Q\left(R^{s}, B, C\right)=\sum_{i=1}^{I} R_{i}^{s} \times \partial_{i}^{B C}
$$

where,

$Q\left(R^{s}, B, C\right) \quad$ The total resident population in segment s which lives within the travel threshold $\mathrm{C}$ of the nearest access point of type B.

Indicator range $0-\sum_{i=1}^{I} R_{i}^{s}$ inclusive.

The number of residents in segment s living in zone $\mathrm{i}$.

$R_{i}^{s}$

$\partial_{i}^{B C}$

An indicator function which assumes the following values: 1 - Unity if zone $i$ is within the travel threshold $\mathrm{C}$ of at least one access point of type B. 0 - Zero otherwise.

The aforementioned expression represents a destination based threshold (cumulative opportunity) accessibility measure, in which the target destinations are the access points which satisfy the specified criteria, and these access points are assumed to be perfect substitutes for each other.

The proportion of the total population within the access-to-public-transport threshold (as proposed in paragraph 4) is:

$$
q\left(R^{s}, B, C\right)=\frac{Q\left(R^{s}, B, C\right)}{\sum_{i=1}^{I} R_{i}^{s}}=\frac{\sum_{i=1}^{I} R_{i}^{s} \times \partial_{i}^{B C}}{\sum_{i=1}^{I} R_{i}^{s}}
$$


where,

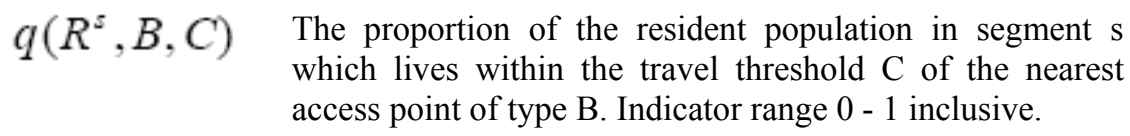

It should be noted that equations 1 and 2 can be applied without an intermediate interpolation and contouring phase with the actual summary indicators calculated through application of equation 1 to each origin point location in turn. It however should be noted that this will most probably produce a result which is not consistent with the use of a GIS based summation phase (i.e. aerial based proportional sum, point count or otherwise).

\subsection{Network measures}

These indicators define the ease of access through the transport network to/from a particular service in terms of journey time, distance, cost or generalised cost, for different departure times or time periods and days of the week.

The methodology in Accession facilitates multi-modal network accessibility assessments through the transport network to/from home and activity locations. It considers the mode(s) of travel, the departure time or the time period, the day of week, the origin locations, and service locations.

As an example consider:

i. Modes of travel: Public transport fixed route / Walk

ii. Departure time: 08:00 hours or am peak

iii. Day of week: Monday-Friday

iv. Origin locations: Home address points/grid

v. Destination locations: Secondary schools

Network accessibility assessment is then undertaken utilising transport and land use/service data items satisfying the above criteria (i, ii, iii, iv, v). Journey time/distance/cost/generalised contours are subsequently produced (preceded by an interpolation stage).

Accessibility reports and queries can be used to determine the total resident population type $\mathrm{s}$ located within the defined journey time/distance/cost/ generalised threshold $\mathrm{C}$ of the nearest activity location of type $\mathrm{K}$ (equation 3 ).

$$
P\left(R^{s}, K, C\right)=\sum_{i=1}^{I} R_{i}^{s} \times \partial_{i}^{K C}
$$

where, 


$$
P\left(R^{s}, K, C\right)
$$

$$
\partial_{i}^{K C}
$$

The total resident population in segment $\mathrm{s}$ which lives within the travel threshold $\mathrm{C}$ of the nearest activity

location of type $\mathrm{K}$. Indicator range

$$
0-\sum_{i=1}^{I} R_{i}^{s}
$$
inclusive.

An indicator function which assumes the following values:

1 - Unity if zone $\mathrm{i}$ is within the travel threshold $\mathrm{C}$ of at least one activity location of type K. 0 - Zero otherwise.

An additional formulation of this expression is the proportion of the relevant population type $\mathrm{s}$ located within the defined journey time/distance/cost/ generalised threshold $\mathrm{C}$ of the nearest activity location of type $\mathrm{K}$ (equation 3):

$$
p\left(R^{s}, K, C\right)=\frac{P\left(R^{s}, K, C\right)}{\sum_{i=1}^{I} R_{i}^{s}}=\frac{\sum_{i=1}^{I} R_{i}^{s} \times \partial_{i}^{K C}}{\sum_{i=1}^{I} R_{i}^{s}}
$$

where,

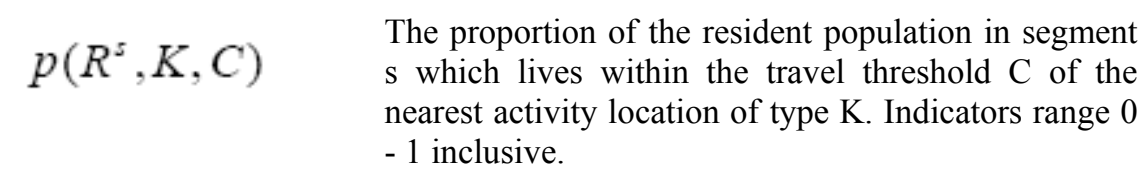

The aforementioned expressions represent a destination based threshold (cumulative opportunity) accessibility measure, in which the target destinations are assumed to be perfect substitutes for each other.

\section{The software tool}

Accession is used to measure accessibility to and through the multi-modal transport system. It calculates indicators of accessibility and uses the indicators and travel time information to describe the quality of the transport system and how it serves the public. Accession produces accessibility contour maps that can be spatially combined with demographic data for an efficient, powerful and to the point accessibility analysis. Accession uses readily available GIS-based network data. These data may include travel times and speeds, restrictions of use and banned turns at intersections. Public transport service data may be imported from a wide range of data formats. Accession accepts census data in practically 
any spatial form. These datasets provide the base for creating the transport network and the accessibility analysis. Accession produces tabular and mapbased indicators for accessibility that may be cross tabulated with other spatial demographic data.

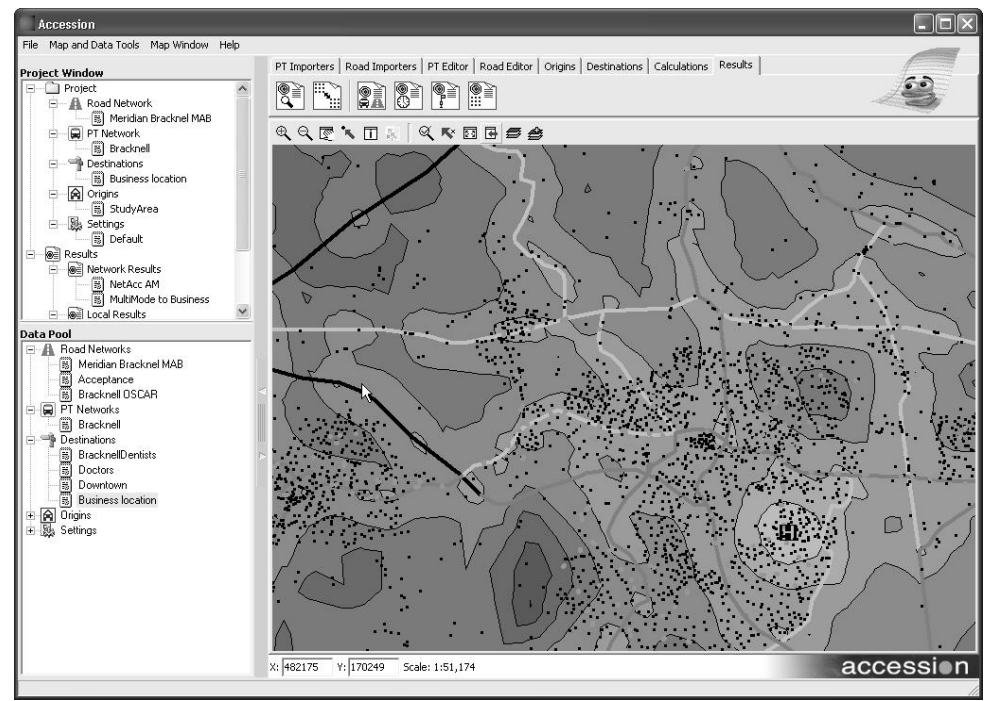

Figure 1: $\quad$ Example of contour based output from Accession.

\subsection{Examples of results}

Accession produces a series of maps and tables to visualise and report accessibility indicators. These include:

\section{Contour maps:}

- resident's access to public transport services

- travel time to locations by mode

- $\quad$ catchment areas for businesses and services

Tables and Indicators:

- travel time and cost matrices by mode

- $\quad$ public transport level of service

- level of accessibility to destinations

The above maps and tables combined with demographic information produces a wealth of information for determining performance indicators for transport services and levels of social exclusion for different population groups. Examples of users of Accession include governmental departments, local authorities, public transport operators, health and education service providers, and land and property developers. These public and private organizations and 
their consultants apply Accession to measure and to improve accessibility for the communities that they serve.

\subsection{Examples applications}

Accession provides the planner with a tool for measuring accessibility to the transport system itself. Other uses of the software include assessment of accessibility to public and private services, location analysis and business and service catchment analysis.

\section{Example 1: Assessment of public transport services}

Accession is used by local transport planning agencies and transport operators to investigate how the public transport system serves the public. Using a fine grid structure, Accession provides indicators of the most convenient stops and stations from any point in the study area. This measure of accessibility is highlighted in contour maps which can be spatially combined with demographic data.

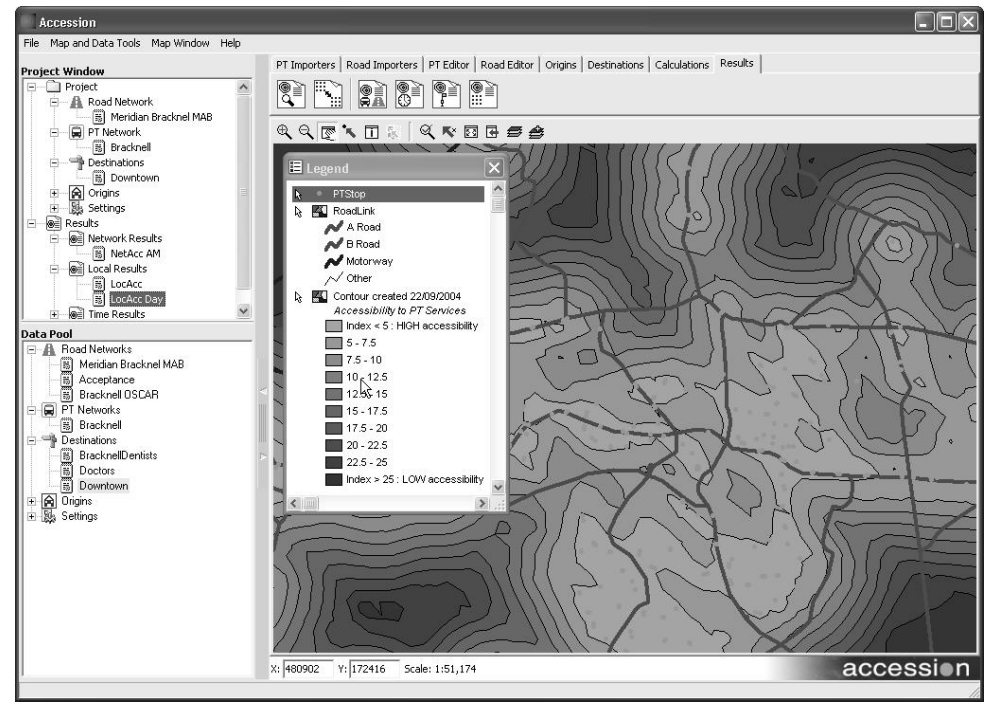

Figure 2: Contour map showing resident's relative accessibility to public transport services.

\section{Example 2: Investigating Accessibility to Health Services}

Accession finds the best routes through the public transport and highway network. For any location, (i.e. for local hospitals), indicators are calculated for any point indicating the barriers of time and cost facing the patients and employees. Accession can model the overall availability of services and can test 'what if' scenarios of changing opening and closing times, locations and transport services to help provide the best overall accessibility. 


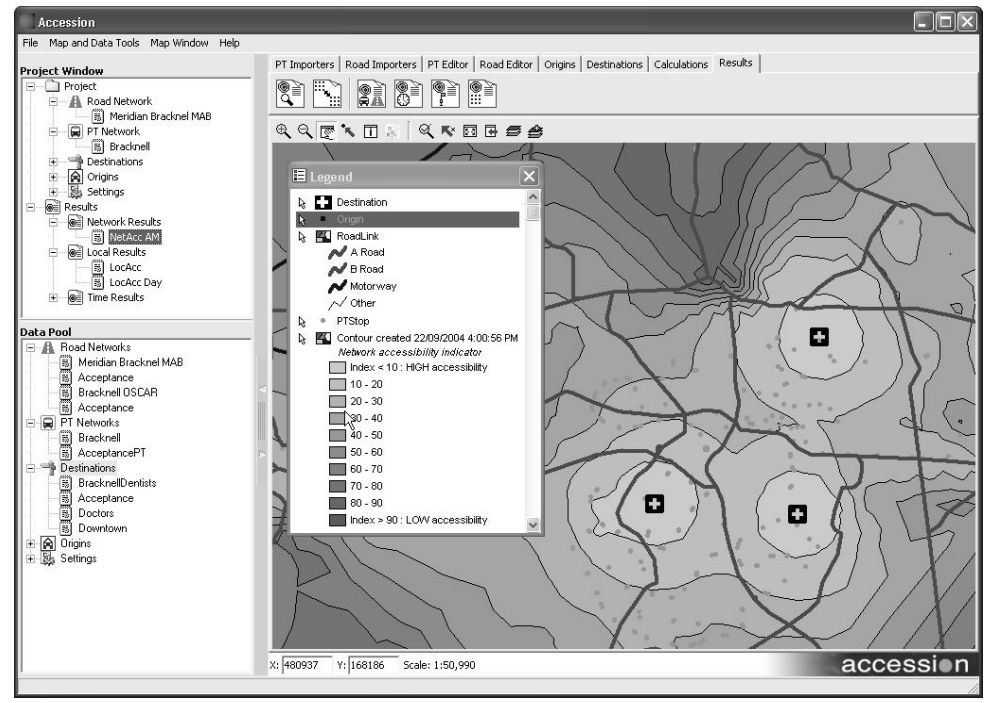

Figure 3: Network-based accessibility of travel time through the public transport system to three hospitals.

\section{Example 3: Investigating social exclusion}

The term 'social exclusion' is used when looking at how different socioeconomic groups are served by essential services that society offers. Accessibility to health services, education, employment and shopping are key measures of social exclusion. Accession overlays accessibility spatially with data for different socio-economic groups in order to pinpoint problem areas. Furthermore, it provides a direct measurement of the ability of different users to reach services and can also identify how accessibility can be improved for groups of people that suffer from social exclusion.

\section{Example 4: Business (re)location and catchment analysis}

Finding the right location for a business can be crucial to its chance of success. For most public oriented businesses, accessibility to the location is a key factor, (i.e. for shopping malls). By producing realistic measures of accessibility to any location, Accession can prove a very efficient tool for location analysis. The accessibility contour information that is produced will be equally useful for catchment analysis and for determining how large areas are realistically covered from alternative business locations.

\subsection{Measuring and highlighting accessibility based on readily available data in a few simple steps}

Accession has a simple to use interface with a built in GIS allowing quick, easy import of standard public transport data formats and digitized road networks. Transportation data can be easily manipulated to allow users to undertake 
accessibility mapping. With a file containing public transport data covering the required study area, creating accessibility maps and getting key statistical information can be done in a few easy steps:

\section{Step 1}

Create a blank project database and save it to your desired location.

\section{Step 2}

Import PT data in the required format from a wide range of available data sources.

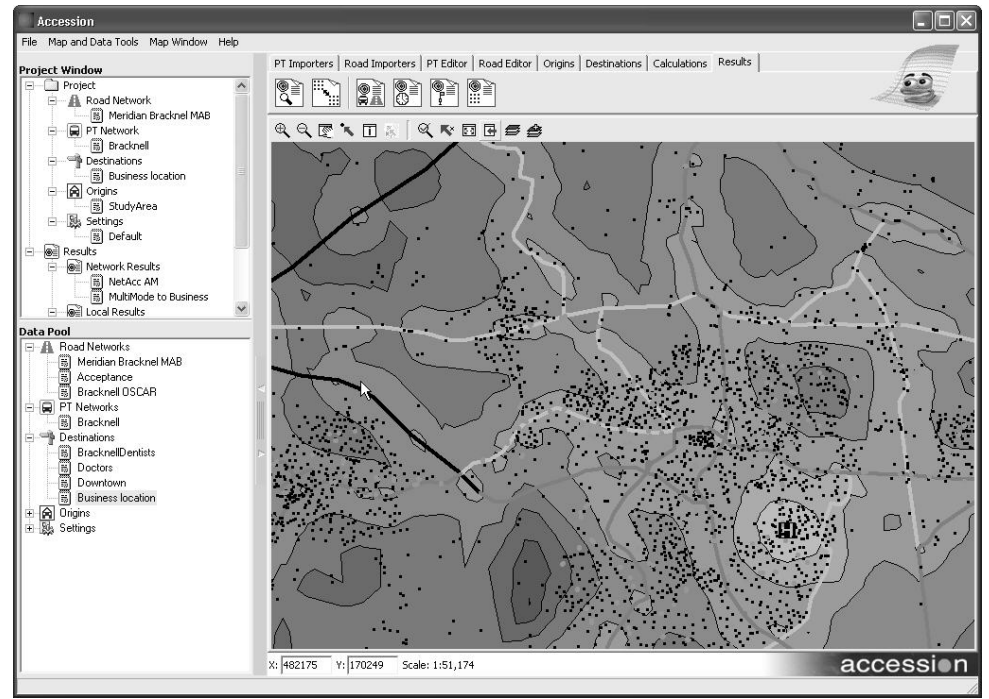

Figure 4: Accessibility to a potential business location by road or public transport.

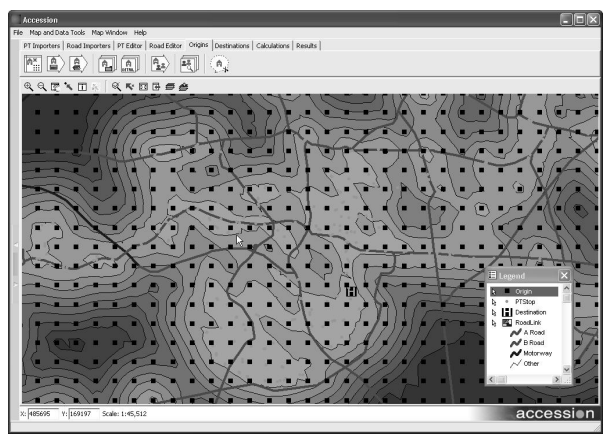

Figure 5: Accession calculates routes from all origin points to the destinations. 


\section{Step 3}

Origin points form the basis for the accessibility mapping. Import an origin data set or create the origin grid with your own defined spacing. Socio-economic data can be assigned to origin points to get the required statistical information required after the accessibility calculation has been undertaken.

\section{Step 4}

Single or multiple destination points can be created. Import a destination data set or create your own destination points.

\section{Step 5}

For the accessibility analysis, select either local (origin based) or network (origin to/from destination) accessibility. Various wizard driven forms are provided for entering the required parameters. Results are stored in the project database.

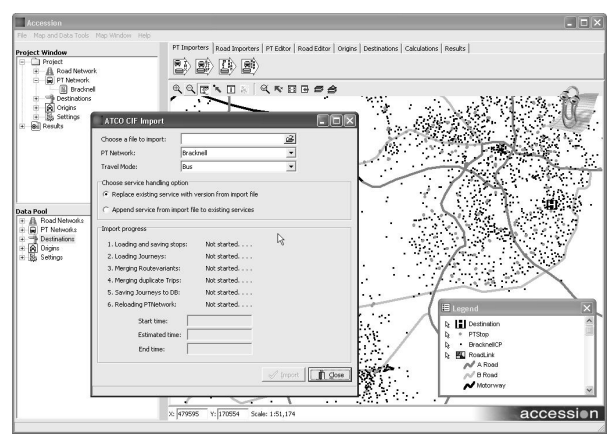

Figure 6: Network-based accessibility of travel time through the public transport system to three hospitals.

\section{Step 6}

Create shaded contours or thematic maps based on the results. Numerous statistical tools are available for comparing with socio economic datasets (i.e. number of people within a stations' catchment area).

The above data created or imported into your project database as well as any result files, contours, thematic maps or reports produced are stored by scenario for future reference.

\subsection{Built in mapping}

Accession has its own GIS map window which negates the need for any third party GIS software. It offers standard navigation tools, full layer control and editing/display functions. Accession is able to link to directories containing mapping data and can use individual map files "on the fly" without translation 
and copying. Any standard GIS formats can be handled, including MapInfo, ESRI or CAD. Images in the map window can be printed, exported or published to the clipboard for use in presentations and reports.

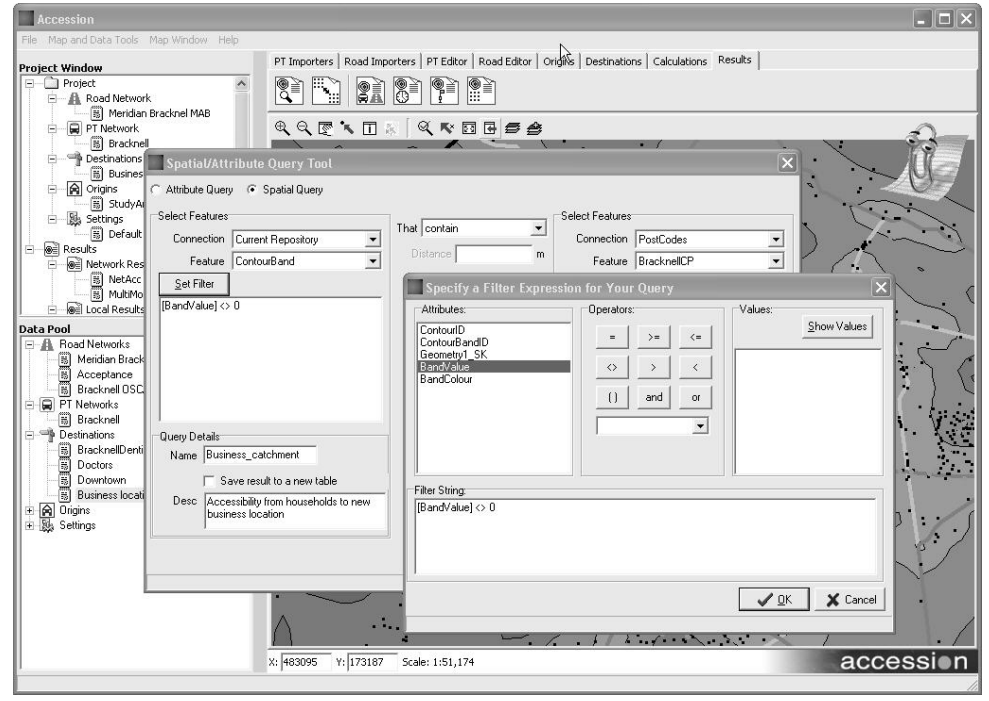

Figure 7: Built in GIS. Accession includes GIS tools such as spatial query functions.

\section{Accession in use}

In the UK, many accessibility audits have been undertaken in the last few years to provide information on travel times by different modes (e.g. car, public transport, cycle and walk) for use with GIS based mapping to relate this to data on population, employment and a range of social criteria. Local Authorities have had to prepare these audits to tackle social exclusion as an essential input to transport and development plans and as a tool for development control.

Accession has also been used by health and education service providers to assess accessibility their consultants have used Accession to plan the location of services and provide transport where access to services from areas of need is poor.

Public transport operators have used Accession in their service planning to assist them respond to objectives and plans set by local authorities.

For the property development sector, Accession has provided information for developers, landowners and their consultants to identify locations suitable for specific types of land-use and appropriate development mixes at individual sites. Accession can also help determine where transport improvements are required to render a site viable for a particular development.

Project examples carried out by MVA Consultancy include: 
- $\quad$ Airdrie - Bathgate Rail Line re-opening where Accession was used to assess the impact on accessibility of possible new stations;

- London Borough Harrow for planning magistrate services;

- The Passport Office for assessing access to Passport offices;

- Ashford Borough Council for town centre planning;

- $\quad$ Portsmouth NHS Trust for site and service development planning;

- Bovis and Heron Homes for preparing sustainable transport proposals for a housing development;

- BT for managing travel and parking and improving access by all travel modes to their Adastral Park site;

- Southern Water for identifying where to locate tertiary sludge treatment facilities;

- Bedfordshire County Council in identifying optimal locations for waste treatment plants;

- Milton Keynes, Birmingham, Solihull, Walsall, Derbyshire, NWRA and Durham in preparing accessibility strategies and plans;

- Brighton International Arena Ltd for accessibility to a leisure complex.

\section{Conclusion}

Accessibility planning is a requirement as part of local transport plans in England. With the methodologies developed in DfT funded projects, where accessibility modelling has been clearly defined and structured, accessibility planning has become an easier task for the local authorities. The software tool Accession has helped these authorities to quickly produce accessibility indicators for access to the transport system itself, to important social and other services and thus being able to address important issues such as social exclusion.

\section{References}

[1] Accessibility planning: Technical Appendix 2. The treatment of local accessibility indicators in Accession, DfT 29 December 2004

[2] DfT web site; Policy Guidance and Research, Accessibility Guidance: Full Guidance, 31 January 2006

[3] MVA Information sheets

[4] Accession web site; www.accessiongis.com; Citilabs May 2007

[5] Accession help system; Version 1.5; Citilabs/MVA February 2006 